\title{
Derivada Topológica Associada ao Modelo de Placas de Reissner-Mindlin
}

\author{
V. Sales, A.A. Novotny e J.E. Muñoz Rivera \\ Laboratório Nacional de Computação Científica LNCC/MCT \\ E-mail: vitors@lncc.br, novotny@lncc.br, rivera@lncc.br
}

Resumo: A derivada topológica mede a sensibilidade de um dado funcional de forma quando uma perturbação singular (furo, inclusão, termo-fonte, etc) infinitesimal é introduzida em um ponto arbitrário do dominio de definição do problema. Esta derivada tem sido aplicada com sucesso no contexto de otimização topológica, problemas inversos, processamento de imagens, modelagem constitutiva multi-escala, mecânica da fratura e modelagem de evolução de dano. Neste trabalho a derivada topológica da energia potencial total associada ao problema de flexão de placas de Reissner-Mindlin é obtida. O modelo matemático associado a placas de ReissnerMindlin é escrito na forma de um sistema de equações diferenciais totalmente acoplado, o que representa a maior dificuldade deste trabalho. De fato, a derivada topológica tem sido apresentada apenas de forma abstrata para essa classe de problemas. Sendo assim, são apresentados argumentos sobre a existência da derivada topológica associada ao modelo em análise, bem como sua forma fechada considerando como perturbação topológica a nucleção de uma inclusão circular. Finalmente, é apresentada uma justificativa matemática completa para as estimativas dos termos remanescentes da expansão assintótica topológica.

Palavras-chave: derivada topológica, modelo de Reissner-Mindlin, análise assintótica

\section{Introdução}

A derivada topológica foi introduzida de maneira rigorosa em [7]. Esta derivada mede a sensibilidade de um dado funcional de forma quando uma perturbação singular (furo, inclusão, termo-fonte, etc) infinitesimal é introduzida em um ponto arbitrário do domínio de definição do problema. A derivada topológica tem sido aplicada com sucesso no contexto de otimização topológica, problemas inversos, processamento de imagens, modelagem constitutiva multi-escala, mecânica da fratura e modelagem de evolução de dano. Neste trabalho a derivada topológica da energia potencial total associada ao problema de flexão de placas de Reissner-Mindlin é obtida. Em particular, são apresentados argumentos sobre a existência da derivada topológica para este modelo, juntamente com estimativas precisas para os resíduos da expansão assintótica topológica. Cabe esclarecer que o modelo matemático associado a placas de Reissner-Mindlin é escrito na forma de um sistema de equações diferenciais totalmente acoplado, o que representa a maior dificuldade deste trabalho. De fato, a derivada topológica tem sido apresentada apenas de forma abstrata para essa classe de problemas [2]. Sendo assim, a derivada topológica associada ao modelo em análise, considerando como perturbação topológica a nucleção de uma inclusão circular, é obtida em sua forma fechada. Como o modelo de flexão de placas de Reissner-Mindlin considera os efeitos de cisalhamento, acredita-se que essa derivada pode ser útil para várias aplicações práticas, permitindo contornar algumas dificuldades numéricas associadas ao modelo de placa de Kirchhoff apresentado em [4, 1], por exemplo.

Para introduzir essas ideias, considera-se um domínio aberto e limitado $\Omega \subset \mathbb{R}^{2}$ sujeito a uma perturbação singular confinada numa pequena região $\omega_{\varepsilon}(\widehat{x})=\widehat{x}+\varepsilon \omega$ de tamanho $\varepsilon$ e centro em $\widehat{x}$. Seja uma função característica associada ao domínio não perturbado, $\Omega$, dada por $\chi=\mathbb{1}_{\Omega}$. Por outro lado, é definida uma função constante por partes associada ao problema 
topologicamente perturbado como $\chi_{\varepsilon}(\widehat{x})=\mathbb{1}_{\Omega}-(1-\gamma) \mathbb{1}_{\omega_{\varepsilon}(\widehat{x})}$, onde $\gamma$ é o contraste. Seja ainda um funcional de forma dado por $\psi\left(\chi_{\varepsilon}(\widehat{x})\right)$ associado ao problema topologicamente perturbado que admite a seguinte expansão assintótica topológica:

$$
\psi\left(\chi_{\varepsilon}(\widehat{x})\right)=\psi(\chi)+f(\varepsilon) \mathcal{T}(\widehat{x})+o(f(\varepsilon)),
$$

onde $\psi(\chi)$ é o funcional de forma associado ao domínio original (não perturbado), $f(\varepsilon)$ é uma função positiva tal que $f(\varepsilon) \rightarrow 0$, quando $\varepsilon \rightarrow 0^{+}$. A função $\widehat{x} \mapsto \mathcal{T}(\widehat{x})$ é chamada de derivada topológica de $\psi$ no ponto $\widehat{x}$, que pode ser vista como uma correção de primeira ordem sobre $\psi(\chi)$ para aproximar $\psi\left(\chi_{\varepsilon}(\widehat{x})\right)$.

\section{Modelo de placa semi-espessa}

Será considerado as hipóteses Cinemáticas do problema de flexão de placa de Reissner-Mindlin $[3,6]$, onde o domínio da placa bidimensional é dado por $\Omega \subset \mathbb{R}^{2}$, com espessura $h>0$ constante, por simplicidade. Será considerado que a placa é submetida a flexão e efeitos de cisalhamento sob as seguintes premissas cinemática: as fibras normais ao plano médio da placa permanecem retas durante o processo de deformação, mas não necessariamente perpendicular ao plano médio, e ainda, estas fibras não sofrem variações no seu comprimento. Consequentemente, as deformações da cortante transversal não são desprezíveis e as deformações normais são nulos. O funcional de forma associado ao problema não perturbado será definido como:

$$
\psi(\chi):=\mathcal{J}(\theta, w)=\frac{1}{2} \int_{\Omega}\left(\mathcal{M}(\theta) \cdot \nabla^{s} \theta+\mathcal{Q}(\theta, w) \cdot(\theta-\nabla w)\right)-\int_{\Gamma_{N_{\theta}}} \bar{m} \cdot \theta+\int_{\Gamma_{N_{w}}} \bar{q} w
$$

onde $\mathcal{M}(\theta)=\mathbb{C} \nabla^{s} \theta$ é o momento fletor $\mathcal{Q}(\theta, w)=\mathbf{K}(\theta-\nabla w)$ é o esforço cisalhante. Os tensores constitutivos $\mathbb{C}$ e $\mathbf{K}$ são considerados isotrópicos e homogêneos, sendo dados por:

$$
\mathbb{C}=\frac{E h^{3}}{1-\nu^{2}}(\nu(\mathbf{I} \otimes \mathbf{I})+(1-\nu) \mathbb{I}) \quad \text { e } \quad \mathbf{K}=\frac{\kappa E h}{2(1+\nu)} \mathbf{I},
$$

onde $E$ é o módulo Young, $\nu$ o coeficiente de Poisson, $\kappa=5 / 6$ o fator de correção do cisalhamento e $h$ a espessura da placa. E ainda, I e II são os tensores identidade de segunda e quarta ordem, respectivamente. Sendo assim a rotação $\theta$ e o deslocamento transversal $w$ são soluções do seguinte problema variacional acoplado: Encontrar o campo $(\theta, w) \in \mathcal{U}$ de tal modo que:

$$
\left\{\begin{array}{rl}
\int_{\Omega}\left(\mathcal{M}(\theta) \cdot \nabla^{s} \eta_{\theta}+\mathcal{Q}(\theta, w) \cdot \eta_{\theta}\right) & =\int_{\Gamma_{N_{\theta}}} \bar{m} \cdot \eta_{\theta} \\
\int_{\Omega} \mathcal{Q}(\theta, w) \cdot \nabla \eta_{w} & =\int_{\Gamma_{N_{w}}} \bar{q} \eta_{w}
\end{array} \quad \forall\left(\eta_{\theta}, \eta_{w}\right) \in \mathcal{V} .\right.
$$

No problema variacional (4), $\mathcal{U}$ é o conjunto das funções admissíveis e $\mathcal{V}$ é o espaço das variações admissíveis, e são dados por:

$$
\begin{aligned}
& \mathcal{U}:=\left\{\left(\varphi_{\theta}, \varphi_{w}\right), \varphi_{\theta} \in \mathbf{H}^{1}(\Omega) \text { e } \varphi_{w} \in H^{1}(\Omega):\left.\varphi_{w}\right|_{\Gamma_{D_{w}}}=\bar{w},\left.\varphi_{\theta}\right|_{\Gamma_{D_{\theta}}}=\bar{\theta}\right\}, \\
& \mathcal{V}:=\left\{\left(\varphi_{\theta}, \varphi_{w}\right), \varphi_{\theta} \in \mathbf{H}^{1}(\Omega) \text { e } \varphi_{w} \in H^{1}(\Omega):\left.\varphi_{w}\right|_{\Gamma_{D_{w}}}=0,\left.\varphi_{\theta}\right|_{\Gamma_{D_{\theta}}}=0\right\}
\end{aligned}
$$

na qual será adotada a notação $\mathbf{H}^{1}(\Omega)=H^{1}(\Omega) \times H^{1}(\Omega)$. E ainda, $\bar{\theta}$ e $\bar{w}$ são as condições de contorno de Dirichlet e $\bar{m}$ e $\bar{q}$ são as condições de contorno de Neumann. Para que o problema esteja bem posto deve-se destacar que $\Gamma_{D_{w}} \cap \Gamma_{N_{w}}=\emptyset$ e $\Gamma_{D_{\theta}} \cap \Gamma_{N_{\theta}}=\emptyset$, onde a medida de $\Gamma_{D_{\theta}}$ e $\Gamma_{D_{w}}$ são diferentes de zero. A fim de garantir a existência e a unicidade da solução de (4) é necessário satisfazer $\Gamma_{D_{w}} \cap \Gamma_{D_{\theta}} \neq \emptyset$ ou a curvatura de $\Gamma_{D_{w}}$ diferente de zero, ou seja, que sua normal não seja constante.

A energia potencial total associada ao problema perturbado pode ser escrito como: 


$$
\begin{aligned}
\psi\left(\chi_{\varepsilon}(\widehat{x})\right):=\mathcal{J}\left(\theta_{\varepsilon}, w_{\varepsilon}\right)=\frac{1}{2} \int_{\Omega} \gamma_{\omega_{\varepsilon}}\left(\mathcal{M}\left(\theta_{\varepsilon}\right) \cdot \nabla^{s} \theta_{\varepsilon}+\mathcal{Q}\left(\theta_{\varepsilon}, w_{\varepsilon}\right) \cdot\left(\theta_{\varepsilon}-\nabla w_{\varepsilon}\right)\right) & -\int_{\Gamma_{N_{\theta}}} \bar{m} \cdot \theta_{\varepsilon}+\int_{\Gamma_{N_{w}}} \bar{q} w_{\varepsilon},
\end{aligned}
$$

onde o parâmetro $\gamma_{\omega_{\varepsilon}}$ é definido como $\gamma_{\omega_{\varepsilon}}:=\left\{1\right.$ se $x \in \Omega \backslash \bar{\omega}_{\varepsilon}$ ou $\gamma$ se $\left.x \in \omega_{\varepsilon}\right\}$. O domínio $\Omega$ será perturbado pela nucleação de uma pequena bola circular $\omega_{\varepsilon}(\widehat{x})=B_{\varepsilon}(\widehat{x})$, onde $\varepsilon$ é o raio e $\widehat{x} \in \Omega$ é o centro. Também será considerado o parâmetro $0<\gamma<\infty$ representando o contraste da propriedade material. As funções $\theta_{\varepsilon}$ e $w_{\varepsilon}$ do funcional (7) são soluções do seguinte problema variacional: Encontrar o campo $\left(\theta_{\varepsilon}, w_{\varepsilon}\right) \in \mathcal{U}_{\varepsilon}$ de tal forma que

$$
\left\{\begin{array}{rl}
\int_{\Omega} \gamma_{\omega_{\varepsilon}}\left(\mathcal{M}\left(\theta_{\varepsilon}\right) \cdot \nabla^{s} \eta_{\theta}+\mathcal{Q}\left(\theta_{\varepsilon}, w_{\varepsilon}\right) \cdot \eta_{\theta}\right) & =\int_{\Gamma_{N_{\theta}}} \bar{m} \cdot \eta_{\theta} \\
\int_{\Omega} \gamma_{\omega_{\varepsilon}} \mathcal{Q}\left(\theta_{\varepsilon}, w_{\varepsilon}\right) \cdot \nabla \eta_{w} & =\int_{\Gamma_{N_{w}}} \bar{q} \eta_{w}
\end{array}, \forall\left(\eta_{\theta}, \eta_{w}\right) \in \mathcal{V}_{\varepsilon}\right.
$$

onde o conjunto $\mathcal{U}_{\varepsilon}$ e o espaço $\mathcal{V}_{\varepsilon}$ são definidos como:

$$
\mathcal{U}_{\varepsilon}:=\left\{\varphi=\left(\varphi_{\theta}, \varphi_{w}\right) \in \mathcal{U}: \llbracket \varphi \rrbracket=0 \text { em } \partial \omega_{\varepsilon}\right\} \quad \text { e } \quad \mathcal{V}_{\varepsilon}:=\left\{\varphi=\left(\varphi_{\theta}, \varphi_{w}\right) \in \mathcal{V}: \llbracket \varphi \rrbracket=0 \text { em } \partial \omega_{\varepsilon}\right\},
$$

em que $\left.\llbracket \varphi \rrbracket\right|_{\partial \omega_{\varepsilon}}:=\left.\varphi\right|_{\Omega \backslash \omega_{\varepsilon}}-\left.\varphi\right|_{\omega_{\varepsilon}}$ representa o salto da função $\varphi$ sobre a fronteira $\partial \omega_{\varepsilon}$.

Lema 1. Seja $\left(\theta_{\varepsilon}, w_{\varepsilon}\right)$ e $(\theta, w)$ soluções do problema perturbado (8) e original (4), respectivamente. Então tem-se que:

$$
\left\|\theta_{\varepsilon}-\theta\right\|_{\mathbf{H}^{1}(\Omega)} \leq C \varepsilon \quad \text { e }\left\|w_{\varepsilon}-w\right\|_{H^{1}(\Omega)} \leq C \varepsilon,
$$

onde $C$ é constante independente do parâmetro $\varepsilon$.

Demonstração. Tomando a diferença entre (4) e (8), tem-se:

$$
\left\{\begin{array}{rl}
\int_{\Omega}\left(\gamma_{\omega_{\varepsilon}} \mathcal{M}\left(\theta_{\varepsilon}\right)-\mathcal{M}(\theta)\right) \cdot \nabla^{s} \eta_{\theta}+\left(\gamma_{\omega_{\varepsilon}} \mathcal{Q}\left(\theta_{\varepsilon}, w_{\varepsilon}\right)-\mathcal{Q}(\theta, w)\right) \cdot \eta_{\theta} & =0 \\
\int_{\Omega}\left(\gamma_{\omega_{\varepsilon}} \mathcal{Q}\left(\theta_{\varepsilon}, w_{\varepsilon}\right)-\mathcal{Q}(\theta, w)\right) \cdot \nabla \eta_{w} & =0
\end{array} \quad, \forall\left(\eta_{\theta}, \eta_{w}\right) \in \mathcal{V}_{\varepsilon}\right.
$$

introduzindo a notação $\widetilde{\theta}_{\varepsilon}=\theta_{\varepsilon}-\theta$ e $\widetilde{w}_{\varepsilon}=w_{\varepsilon}-w$, a equação variacional pode ser escrita como

$$
\left\{\begin{aligned}
\int_{\Omega} \gamma_{\omega_{\varepsilon}} \mathcal{M}\left(\widetilde{\theta}_{\varepsilon}\right) \cdot \nabla^{s} \eta_{\theta}+\gamma_{\omega_{\varepsilon}} \mathcal{Q}\left(\widetilde{\theta}_{\varepsilon}, \widetilde{w}_{\varepsilon}\right) \cdot \eta_{\theta} & =(1-\gamma) \int_{B_{\varepsilon}} \mathcal{M}(\theta) \cdot \nabla^{s} \eta_{\theta}+\mathcal{Q}(\theta, w) \cdot \eta_{\theta} \\
\int_{\Omega} \gamma_{\omega_{\varepsilon}} \mathcal{Q}\left(\widetilde{\theta}_{\varepsilon}, \widetilde{w}_{\varepsilon}\right) \cdot \nabla \eta_{w} & =(1-\gamma) \int_{B_{\varepsilon}} \mathcal{Q}(\theta, w) \cdot \nabla \eta_{w}
\end{aligned}\right.
$$

onde os termos $\pm \int_{B_{\varepsilon}} \gamma \mathcal{M}(\theta) \cdot \nabla^{s} \eta_{\theta}+\gamma \mathcal{Q}(\theta, w) \cdot \eta_{\theta} \quad$ e $\quad \pm \int_{B_{\varepsilon}} \gamma \mathcal{Q}(\theta, w) \cdot \nabla \eta_{w}$, foram somados à $(11)_{1}$ e $(11)_{2}$, respectivamente. Agora tomando $\eta_{\theta}=\widetilde{\theta}_{\varepsilon}$ e $\eta_{w}=\widetilde{w}_{\varepsilon}$ como função teste nas equações (12) tem-se as seguintes equações:

$$
\left\{\begin{aligned}
\int_{\Omega} \gamma_{\omega_{\varepsilon}} \mathcal{M}\left(\widetilde{\theta}_{\varepsilon}\right) \cdot \nabla^{s} \widetilde{\theta}_{\varepsilon}+\gamma_{\omega_{\varepsilon}} \mathcal{Q}\left(\widetilde{\theta}_{\varepsilon}, \widetilde{w}_{\varepsilon}\right) \cdot \widetilde{\theta}_{\varepsilon} & =(1-\gamma) \int_{B_{\varepsilon}} \mathcal{M}(\theta) \cdot \nabla^{s} \widetilde{\theta}_{\varepsilon}+\mathcal{Q}(\theta, w) \cdot \widetilde{\theta}_{\varepsilon} \\
\int_{\Omega} \gamma_{\omega_{\varepsilon}} \mathcal{Q}\left(\widetilde{\theta}_{\varepsilon}, \widetilde{w}_{\varepsilon}\right) \cdot \nabla \widetilde{w}_{\varepsilon} & =(1-\gamma) \int_{B_{\varepsilon}} \mathcal{Q}(\theta, w) \cdot \nabla \widetilde{w}_{\varepsilon}
\end{aligned}\right.
$$

Aplicando a desigualdade de Cauchy-Schwarz e utilizando a regularidade elíptica de $\theta$ e $w$ tem-se

$$
\left\{\begin{aligned}
& \int_{\Omega} \gamma_{\omega_{\varepsilon}} \mathcal{M}\left(\widetilde{\theta}_{\varepsilon}\right) \cdot \nabla^{s} \widetilde{\theta}_{\varepsilon}+\gamma_{\omega_{\varepsilon}} \mathcal{Q}\left(\widetilde{\theta}_{\varepsilon}, \widetilde{w}_{\varepsilon}\right) \cdot \widetilde{\theta}_{\varepsilon} \leq C_{1} \varepsilon\left\|\widetilde{\theta}_{\varepsilon}\right\|_{\mathbf{H}^{1}\left(B_{\varepsilon}\right)} \\
& \int_{\Omega} \gamma_{\omega_{\varepsilon}} \mathcal{Q}\left(\widetilde{\theta}_{\varepsilon}, \widetilde{w}_{\varepsilon}\right) \cdot \nabla \widetilde{w}_{\varepsilon} \leq C_{2} \varepsilon\left\|\widetilde{w}_{\varepsilon}\right\|_{H^{1}\left(B_{\varepsilon}\right)}
\end{aligned}\right.
$$


Então, após de subtrair a segunda desigualdade da primeira, e usando a desigualdade triangular tem-se:

$$
\int_{\Omega} \gamma_{\omega_{\varepsilon}} \mathcal{M}\left(\widetilde{\theta}_{\varepsilon}\right) \cdot \nabla^{s} \widetilde{\theta}_{\varepsilon}+\gamma_{\omega_{\varepsilon}} \mathcal{Q}\left(\widetilde{\theta}_{\varepsilon}, \widetilde{w}_{\varepsilon}\right) \cdot\left(\widetilde{\theta}_{\varepsilon}-\nabla \widetilde{w}_{\varepsilon}\right) \leq C_{3} \varepsilon\left(\left\|\widetilde{\theta}_{\varepsilon}\right\|_{\mathbf{H}^{1}\left(B_{\varepsilon}\right)}+\left\|\widetilde{w}_{\varepsilon}\right\|_{H^{1}\left(B_{\varepsilon}\right)}\right) .
$$

utilizando a coercividade na forma bilinear no lado esquerdo da expressão acima, pode-se escrever

$$
\alpha\left(\left\|\widetilde{\theta}_{\varepsilon}\right\|_{\mathbf{H}^{1}(\Omega)}^{2}+\left\|\widetilde{w}_{\varepsilon}\right\|_{H^{1}(\Omega)}^{2}\right) \leq \int_{\Omega} \gamma_{\omega_{\varepsilon}} \mathcal{M}\left(\widetilde{\theta}_{\varepsilon}\right) \cdot \nabla^{s} \widetilde{\theta}_{\varepsilon}+\gamma_{\omega_{\varepsilon}} \mathcal{Q}\left(\widetilde{\theta}_{\varepsilon}, \widetilde{w}_{\varepsilon}\right) \cdot\left(\widetilde{\theta}_{\varepsilon}-\nabla \widetilde{w}_{\varepsilon}\right),
$$

Portanto,

$$
\begin{aligned}
C_{3} \varepsilon\left(\left\|\widetilde{\theta}_{\varepsilon}\right\|_{\mathbf{H}^{1}(\Omega)}+\left\|\widetilde{w}_{\varepsilon}\right\|_{H^{1}(\Omega)}\right) & \geq \alpha\left(\left\|\widetilde{\theta}_{\varepsilon}\right\|_{\mathbf{H}^{1}(\Omega)}^{2}+\left\|\widetilde{w}_{\varepsilon}\right\|_{H^{1}(\Omega)}^{2}\right) \\
& \geq \frac{\alpha}{2}\left(\left\|\widetilde{\theta}_{\varepsilon}\right\|_{\mathbf{H}^{1}(\Omega)}+\left\|\widetilde{w}_{\varepsilon}\right\|_{H^{1}(\Omega)}\right)^{2} .
\end{aligned}
$$

Dada a constante $C=2 C_{3} \alpha^{-1}$ independente do parâmetro $\varepsilon$, tem-se

$$
\left\|\widetilde{\theta}_{\varepsilon}\right\|_{\mathbf{H}^{1}(\Omega)}+\left\|\widetilde{w}_{\varepsilon}\right\|_{H^{1}(\Omega)} \leq C \varepsilon,
$$

\section{Forma explicita da Derivada topológica}

Tomando $\eta_{w}=w_{\varepsilon}-w$ e $\eta_{\theta}=\theta_{\varepsilon}-\theta$ como função teste em (4) e (8) e substituindo nos funcionais de forma (2) e (7) e temos que a diferença entre os funcionais é dada por,

$$
\psi\left(\chi_{\varepsilon}(\widehat{x})\right)-\psi(\chi)=-\frac{1-\gamma}{2} \int_{B_{\varepsilon}}\left(\mathcal{M}(\theta) \cdot \nabla^{s} \theta_{\varepsilon}+\mathcal{Q}(\theta, w) \cdot\left(\theta_{\varepsilon}-\nabla w_{\varepsilon}\right)\right)
$$

onde usou-se a definição para o contraste $\gamma_{\omega_{\varepsilon}}$. Note que a diferença acima é dada por uma integral concentrada na bola $B_{\varepsilon}$. Portanto, é necessário conhecer o comportamente das funções $\theta_{\varepsilon}$ e $w_{\varepsilon}$ quando $\varepsilon \rightarrow 0$ na região da perturbação $B_{\varepsilon}$. Então considera-se os seguintes ansätzes (expansões em potências de $\varepsilon$ ) para as soluções $\theta_{\varepsilon}$ e $w_{\varepsilon}$ do problema variacional (8)

$$
\theta_{\varepsilon}(x)=\theta(x)+\varepsilon \phi(x / \varepsilon)+\widetilde{\theta}_{\varepsilon}(x) \quad \text { e } \quad w_{\varepsilon}(x)=w(x)+\varepsilon z(x / \varepsilon)+\widetilde{w}_{\varepsilon}(x) .
$$

onde as funções $\theta$ e $w$ são as soluções para o problema do valor limite não perturbado (4), enquanto que as funções $\varphi$ e $z$ são as soluções dos problemas exteriores e $\widetilde{\theta}_{\varepsilon}$ e $\widetilde{w}_{\varepsilon}$ são os resíduos. Em particular, a partir de uma alteração de variáveis na forma $x=\varepsilon y$, função $\varphi$ e $z$ são soluções dos seguintes problemas de valor de contorno

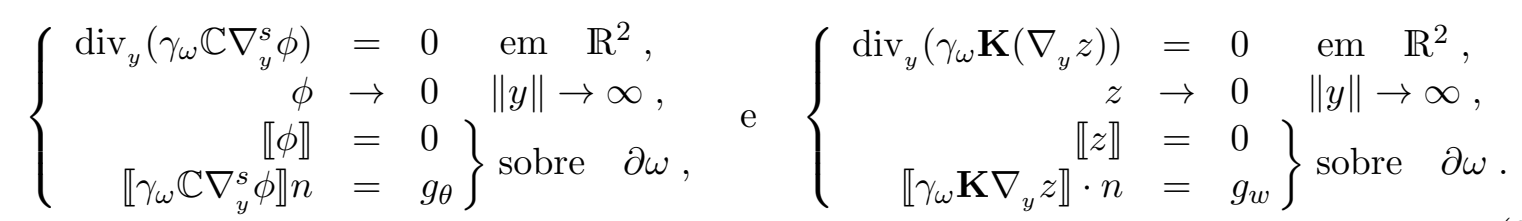

onde $g_{\theta}=-(1-\gamma) \mathcal{M}(\theta(\widehat{x})) n$ e $g_{w}=(1-\gamma) \mathcal{Q}(\theta, w)(\widehat{x}) \cdot n$. O resíduo $\left(\widetilde{\theta}_{\varepsilon}, \widetilde{w}_{\varepsilon}\right)$ é solução do seguinte problema contorno. Este é construído de modo a compensar as discrepâncias geradas por $\phi$ e $z$ e pelos termos de ordem superior da expansão em series Taylor de $\mathcal{M}(\theta(x))$ e $\mathcal{Q}(\theta(x), w(x))$ em torno do ponto $\widehat{x} \in \Omega$, então,

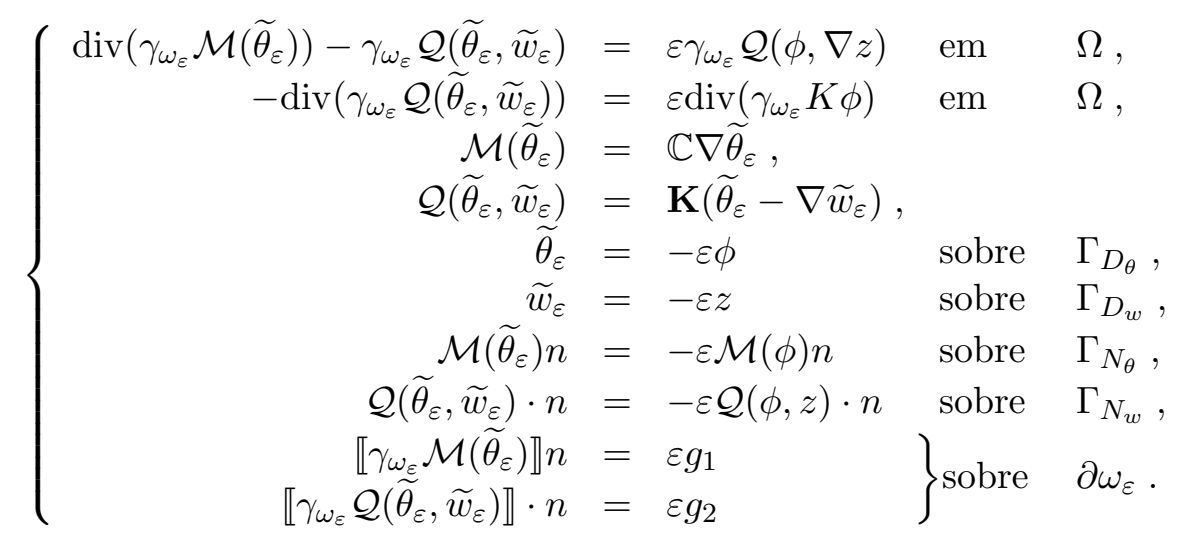


onde $g_{1}=-(1-\gamma)\left(\nabla \mathcal{M}(\theta)\left(\xi_{1}\right) n\right) n$ e $g_{2}=-(1-\gamma)\left(\nabla \mathcal{Q}(\theta, w)\left(\xi_{2}\right) n-\mathbf{K} \phi\right) \cdot n$, com $\xi_{1}$ e $\xi_{2}$ sendo pontos entre $x$ e $\widehat{x}$.

Lema 2. Seja $\left(\widetilde{\theta}_{\varepsilon}, \widetilde{w}_{\varepsilon}\right)$ solução de (23). Então, as seguintes estimativas são válidas:

$$
\left\|\widetilde{\theta}_{\varepsilon}\right\|_{\mathbf{H}^{1}(\Omega)}+\left\|\widetilde{w}_{\varepsilon}\right\|_{H^{1}(\Omega)} \leq C \varepsilon,
$$

onde a constante Cé independente do parâmetro $\varepsilon$.

Demonstração. Depois de aplicar a desigualdade triangular nas expressões em (21), obtém-se

$$
\begin{array}{r}
\left|\widetilde{\theta}_{\varepsilon}(x)\right|_{\mathbf{H}^{1}(\Omega)} \leq\left|\theta_{\varepsilon}(x)-\theta(x)\right|_{\mathbf{H}^{1}(\Omega)}+\varepsilon|\phi(x / \varepsilon)|_{\mathbf{H}^{1}(\Omega)}, \\
\left|\widetilde{w}_{\varepsilon}(x)\right|_{H^{1}(\Omega)} \leq\left|w_{\varepsilon}(x)-w(x)\right|_{H^{1}(\Omega)}+\varepsilon|z(x / \varepsilon)|_{H^{1}(\Omega)} .
\end{array}
$$

Com o Lema 1 é dado as estimativas em (10), então tem-se

$$
\begin{aligned}
\left\|\widetilde{\theta}_{\varepsilon}(x)\right\|_{\mathbf{H}^{1}(\Omega)} & \leq C \varepsilon+\varepsilon|\phi(y)|_{\mathbf{H}^{1}\left(\mathbb{R}^{2}\right)} \leq \bar{C} \varepsilon, \\
\left\|\widetilde{w}_{\varepsilon}(x)\right\|_{H^{1}(\Omega)} & \leq C \varepsilon+\varepsilon|z(y)|_{H^{1}\left(\mathbb{R}^{2}\right)} \leq \widetilde{C} \varepsilon,
\end{aligned}
$$

onde usou-se o fato que as funções $\phi(y)$ e $z(y)$ são independentes de $\varepsilon$, com $x=\varepsilon y$ e no lado esquerdo da desigualdade utilizou-se a coercividade.

Substituindo as estimativas em (21) em (20) obtém-se o seguinte resultado,

$$
\begin{aligned}
\psi\left(\chi_{\varepsilon}(\widehat{x})\right)-\psi(\chi)=-\frac{1-\gamma}{2} \int_{B_{\varepsilon}}\left(\left(\mathcal{M}(\theta) \cdot \nabla^{s} \theta+\mathcal{Q}(\theta, w) \cdot(\theta-\nabla w)\right)(\widehat{x})\right. & \\
& \left.+\varepsilon \mathcal{M}(\theta)(\widehat{x}) \cdot \nabla^{s} \phi-\varepsilon \mathcal{Q}(\theta, w)(\widehat{x}) \cdot \nabla z\right)+\mathcal{E}(\varepsilon),
\end{aligned}
$$

onde $\mathcal{E}(\varepsilon)=\sum_{i=1}^{6} \mathcal{E}_{i}(\varepsilon)=o\left(\varepsilon^{2}\right)$ como pode ser visto na Seção 4 , com:

$$
\begin{aligned}
& \mathcal{E}_{1}(\varepsilon)=-\frac{1-\gamma}{2} \int_{B_{\varepsilon}}\left(\mathcal{M}(\theta) \cdot \nabla^{s} \widetilde{\theta}_{\varepsilon}+\mathcal{Q}(\theta, w) \cdot\left(\widetilde{\theta}_{\varepsilon}-\nabla \widetilde{w}_{\varepsilon}\right)\right) \\
& \mathcal{E}_{2}(\varepsilon)=-\frac{1-\gamma}{2} \int_{B_{\varepsilon}}\left(\mathcal{M}(\theta) \cdot \nabla^{s} \theta-\left(\mathcal{M}(\theta) \cdot \nabla^{s} \theta\right)(\widehat{x})\right) \\
& \mathcal{E}_{3}(\varepsilon)=-\varepsilon \frac{1-\gamma}{2} \int_{B_{\varepsilon}}\left(\nabla^{s} \phi \cdot(\mathcal{M}(\theta)-\mathcal{M}(\theta)(\widehat{x}))\right) \\
& \mathcal{E}_{4}(\varepsilon)=-\frac{1-\gamma}{2} \int_{B_{\varepsilon}}(\mathcal{Q}(\theta, w) \cdot(\theta-\nabla w)-(\mathcal{Q}(\theta, w) \cdot(\theta-\nabla w))(\widehat{x})), \\
& \mathcal{E}_{5}(\varepsilon)=-\varepsilon \frac{1-\gamma}{2} \int_{B_{\varepsilon}}(\phi-\nabla z) \cdot(\mathcal{Q}(\theta, w)-\mathcal{Q}(\theta, w)(\widehat{x})) \\
& \mathcal{E}_{6}(\varepsilon)=-\varepsilon \frac{1-\gamma}{2} \int_{B_{\varepsilon}} \phi \cdot \mathcal{Q}(\theta, w)(\widehat{x}) .
\end{aligned}
$$

dada a mudança de variável $x=\varepsilon y$ e a estimativa $\mathcal{E}(\varepsilon)=o\left(\varepsilon^{2}\right)$, escreve-se $(29)$ como:

$$
\begin{aligned}
\psi\left(\chi_{\varepsilon}(\widehat{x})\right)-\psi(\chi)=-\varepsilon^{2} \frac{1-\gamma}{2} \int_{B_{1}} & \left(\left(\mathcal{M}(\theta) \cdot \nabla^{s} \theta+\mathcal{Q}(\theta, w) \cdot(\theta-\nabla w)\right)(\widehat{x})\right. \\
& +\nabla^{s} \theta(\widehat{x}) \cdot \mathbb{C} \nabla_{y}^{s} \phi(y)-(\theta-\nabla w)(\widehat{x}) \cdot \mathbf{K} \nabla_{y} z(y)+o\left(\varepsilon^{2}\right),
\end{aligned}
$$

As soluções dos problemas em (22) têm exatamente a mesma estrutura do que os problemas de Navier e Laplace. Além disso, as soluções para o caso particular onde a perturbação é de 
forma circular são conhecidas, para maiores detalhes ver o livro de Novotny \& Sokołowski [5]. Utilizando o teorema de Eshelby, pode-se escrever para dentro da inclusão, o seguinte:

$$
\left.\mathbb{C} \nabla_{y}^{s} \phi(y)\right|_{B_{1}}=\mathbb{T} \mathcal{M}(\theta)(\widehat{x}) \quad \text { e }\left.\quad \mathbf{K} \nabla_{y} z(y)\right|_{B_{1}}=\mathbf{T} \mathcal{Q}(\theta, w)(\widehat{x})
$$

onde $\mathbb{T}$ e $\mathbf{T}$ são tensores isotrópicos de quarta e segunda ordem, e são dados por:

$$
\mathbb{T}=\frac{1}{2} \frac{1-\gamma}{1+\gamma \alpha_{2}}\left(2 \alpha_{2} \mathbb{I}+\frac{\alpha_{1}-\alpha_{2}}{1+\gamma \alpha_{1}} \mathbf{I} \otimes \mathbf{I}\right) \quad \text { e } \quad \mathbf{T}=\frac{1-\gamma}{1+\gamma} \mathbf{I}
$$

Agora, substituindo as igualdades dadas por (37) na diferença (36) tem-se,

$$
\psi\left(\chi_{\varepsilon}(\widehat{x})\right)-\psi(\chi)=\pi \varepsilon^{2}\left(\mathbb{P} \mathcal{M}(\theta(\widehat{x})) \cdot \nabla^{s} \theta(\widehat{x})+\mathbf{P} \mathcal{Q}(\theta(\widehat{x}), w(\widehat{x})) \cdot(\theta(\widehat{x})-\nabla w(\widehat{x}))\right)+o\left(\varepsilon^{2}\right) .
$$

Portanto a derivada topológica $\mathcal{T}(\widehat{x})$ será

$$
\mathcal{T}(\widehat{x})=\mathbb{P} \mathcal{M}(\theta(\widehat{x})) \cdot \nabla^{s} \theta(\widehat{x})+\mathbf{P} \mathcal{Q}(\theta(\widehat{x}), w(\widehat{x})) \cdot(\theta(\widehat{x})-\nabla w(\widehat{x})),
$$

onde $f(\varepsilon)=\pi \varepsilon^{2}$. E ainda a matriz de polarização $d \mathbb{P}$ é associada ao efeito de flexão enquanto que $\mathbf{P}$ é associada aos efeito cisalhante e são dadas por

$$
\mathbb{P}=-\frac{1-\gamma}{2}(\mathbb{I}+\mathbb{T}) \quad \text { e } \quad \mathbf{P}=-\frac{1-\gamma}{2}(\mathbf{I}+\mathbf{T})
$$

Finalmente, os coeficientes $\alpha_{1}=(1+\nu) /(1-\nu)$ e $\alpha_{2}=(3+\nu) /(1+\nu)$.

\section{Estimativas para os termos remanescentes}

Nesta seção, será estimado o resíduo $\mathcal{E}$ da expansão assintótica (29).

Considerando $\mathcal{E}_{1}(\varepsilon)$ dado por (30) e a notação $M_{1}=\mathcal{M}(\theta)$ e $Q_{1}=\mathcal{Q}(\theta, w)$, então

$$
\begin{aligned}
& \mathcal{E}_{1}(\varepsilon)=-\frac{1-\gamma}{2} \int_{B_{\varepsilon}}\left(M_{1}(x) \pm M_{1}(\widehat{x})\right) \cdot \nabla^{s} \widetilde{\theta}_{\varepsilon}+\left(Q_{1}(x) \pm Q_{1}(\widehat{x})\right) \cdot\left(\widetilde{\theta}_{\varepsilon}-\nabla \widetilde{w}_{\varepsilon}\right) \\
& \leq C_{1}\left(\left\|M_{1}(x)-M_{1}(\widehat{x})\right\|_{\mathbf{L}^{2}\left(B_{\varepsilon}\right)}\left\|\nabla^{s} \widetilde{\theta}_{\varepsilon}\right\|_{\mathbf{L}^{2}\left(B_{\varepsilon}\right)}+\left\|M_{1}(\widehat{x})\right\|_{\mathbf{L}^{2}\left(B_{\varepsilon}\right)}\left\|\nabla^{s} \widetilde{\theta}_{\varepsilon}\right\|_{\mathbf{L}^{2}\left(B_{\varepsilon}\right)}\right. \\
&\left.\quad+\left\|Q_{1}(x)-Q_{1}(\widehat{x})\right\|_{\mathbf{L}^{2}\left(B_{\varepsilon}\right)}\left\|\widetilde{\theta}_{\varepsilon}-\nabla \widetilde{w}_{\varepsilon}\right\|_{\mathbf{L}^{2}\left(B_{\varepsilon}\right)}+\left\|Q_{1}(\widehat{x})\right\|_{\mathbf{L}^{2}\left(B_{\varepsilon}\right)}\left\|\widetilde{\theta}_{\varepsilon}-\nabla \widetilde{w}_{\varepsilon}\right\|_{\mathbf{L}^{2}\left(B_{\varepsilon}\right)}\right) .
\end{aligned}
$$

Para regularidade elíptica das funções $\theta$ e $w$, tem-se $\left\|M_{1}(x)-M_{1}(\widehat{x})\right\| \leq \bar{c}_{1}\|x-\widehat{x}\|$ e $\| Q_{1}(x)-$ $Q_{1}(\widehat{x})\left\|\leq \widetilde{c}_{1}\right\| x-\widehat{x} \|$, onde $\widetilde{c}_{1}$ e $\bar{c}_{1}$ são constantes independentes do parâmetro $\varepsilon$, então

$$
\begin{aligned}
\mathcal{E}_{1}(\varepsilon) \leq C_{2}\left(\|x-\widehat{x}\|_{\mathbf{L}^{2}\left(B_{\varepsilon}\right)}\left\|\nabla^{s} \widetilde{\theta}_{\varepsilon}\right\|_{\mathbf{L}^{2}\left(B_{\varepsilon}\right)}+\left\|M_{1}(\widehat{x})\right\|_{\mathbf{L}^{2}\left(B_{\varepsilon}\right)}\left\|\nabla^{s} \widetilde{\theta}_{\varepsilon}\right\|_{\mathbf{L}^{2}\left(B_{\varepsilon}\right)}\right. \\
\left.\quad+\|x-\widehat{x}\|_{\mathbf{L}^{2}\left(B_{\varepsilon}\right)}\left\|\widetilde{\theta}_{\varepsilon}-\nabla \widetilde{w}_{\varepsilon}\right\|_{\mathbf{L}^{2}\left(B_{\varepsilon}\right)}+\left\|Q_{1}(\widehat{x})\right\|_{\mathbf{L}^{2}\left(B_{\varepsilon}\right)}\left\|\widetilde{\theta}_{\varepsilon}-\nabla \widetilde{w}_{\varepsilon}\right\|_{\mathbf{L}^{2}\left(B_{\varepsilon}\right)}\right) \\
\leq C_{3} \varepsilon^{2}\left(\left\|\nabla^{s} \widetilde{\theta}_{\varepsilon}\right\|_{\mathbf{L}^{2}\left(B_{\varepsilon}\right)}+\left\|\widetilde{\theta}_{\varepsilon}-\nabla \widetilde{w}_{\varepsilon}\right\|_{\mathbf{L}^{2}\left(B_{\varepsilon}\right)}\right)
\end{aligned}
$$

onde usou-se o fato que $Q_{1}(\widehat{x})$ e $M_{1}(\widehat{x})$ são tensores uniformes. Utilizando a desigualdade triangular Juntamente com o Lema 2, finalmente obtemos

$$
\begin{aligned}
\mathcal{E}_{1}(\varepsilon) & \leq C_{3} \varepsilon^{2}\left(\left\|\nabla^{s} \widetilde{\theta}_{\varepsilon}\right\|_{\mathbf{L}^{2}(\Omega)}+\left\|\widetilde{\theta}_{\varepsilon}\right\|_{\mathbf{L}^{2}(\Omega)}+\left\|\nabla \widetilde{w}_{\varepsilon}\right\|_{L^{2}(\Omega)}\right) \\
& \leq C_{3} \varepsilon^{2}\left(\left\|\widetilde{\theta}_{\varepsilon}\right\|_{\mathbf{H}^{1}(\Omega)}+\left\|\widetilde{w}_{\varepsilon}\right\|_{H^{1}(\Omega)}\right)=o\left(\varepsilon^{2}\right) .
\end{aligned}
$$

Sejam os resíduos $\mathcal{E}_{2}(\varepsilon)$ e $\mathcal{E}_{3}(\varepsilon)$ dados respectivamente por (31) e (32). Introduzindo as notações $h_{2}=\mathcal{M}(\theta) \cdot \nabla^{s} \theta$ e $h_{3}=\mathcal{Q}(\theta, w) \cdot(\theta-\nabla w)$ e ainda considerando a regularidade elíptica 
das funções $\theta$ e $w$, tem-se $\left\|h_{i}(x)-h_{i}(\widehat{x})\right\| \leq c_{i}\|x-\widehat{x}\|$, com $i=2,3$, onde $c_{i}$ são constantes independentes de $\varepsilon$. Portanto,

$$
\mathcal{E}_{i}(\varepsilon)=-\frac{1-\gamma}{2} \int_{B_{\varepsilon}}\left(h_{i}(x)-h_{i}(\widehat{x})\right) \leq C_{i} \int_{B_{\varepsilon}}\|x-\widehat{x}\|=o\left(\varepsilon^{2}\right) .
$$

Introduzindo as notações $G_{4}=\nabla^{s} \phi, H_{4}=\mathcal{M}(\theta)$ em $(33)$ e $G_{5}=\phi-\nabla z, H_{5}=\mathcal{Q}(\theta, w)$ em (34), tem-se $\left\|H_{i}(x)-H_{i}(\widehat{x})\right\| \leq c_{i}\|x-\widehat{x}\|$ para $i=4,5$, onde $c_{i}$ são constantes independentes de $\varepsilon$. Cabe esclarecer que foi utilizada novamente a regularidade elíptica das funções $\theta$ e $w$. Então tem-se as seguintes estimativas para os resíduos $\mathcal{E}_{i}(\varepsilon)$, com $i=4,5$,

$$
\mathcal{E}_{i}(\varepsilon) \leq \varepsilon C_{0}\left\|G_{i}\right\|_{L^{2}\left(B_{\varepsilon}\right)}\left\|H_{i}(x)-H_{i}(\widehat{x})\right\|_{L^{2}\left(B_{\varepsilon}\right)} \leq \varepsilon C_{i}\left\|G_{i}\right\|_{L^{2}\left(B_{\varepsilon}\right)}\|x-\widehat{x}\|_{L^{2}\left(B_{\varepsilon}\right)}=o\left(\varepsilon^{3}\right) .
$$

Finalmente, introduzindo a mudança de variável $x=\varepsilon y$, o resíduo $\mathcal{E}_{6}(\varepsilon)$ dado por (35) pode ser escrito como

$$
\mathcal{E}_{6}(\varepsilon)=-\varepsilon^{3} \frac{1-\gamma}{2} \int_{B_{1}} \phi(y) \cdot \mathcal{Q}(\theta, w)(\widehat{x})=o\left(\varepsilon^{2}\right)
$$

\section{Conclusão}

Neste trabalho a derivada topológica da energia potencial total associada ao problema de flexão de placa de Reissner-Mindlin foi calculada de forma explícita considerando como perturbação topológica a nucleação de inclusões circulares. Esse modelo é representado por um sistema de equações diferenciais parciais totalmente acoplado. Para contornar a dificuldade oriunda desse acoplamento, foi introduzido um conjunto de ansätzes apropriado que conduziu a dois problemas exteriores completamente desacoplados. Como esses problemas admitem soluções explícitas, a derivada topológica foi obtida em sua forma fechada, o que representa a principal contribuição deste trabalho. Além disso, a existência da derivada topológica foi provada e as estimativas para os resíduos da expansão assintótica topológica foram obtidas de forma rigorosa. O resultado obtido pode ser utilizado na otimização topológica de estruturas submetidas a efeitos de flexão no plano e cisalhamento transversal, permitindo contornar algumas dificuldades numéricas associadas ao modelo de flexão de placas de Kirchhoff relatadas em [4].

\section{Referências}

[1] S. Amstutz. A penalty method for topology optimization subject to a pointwise state constraint. ESAIM: Control, Optimisation and Calculus of Variations, 16(03):523-544, 2010.

[2] G. Cardone, S.A. Nazarov, and J. Sokołowski. Asymptotic analysis, polarization matrices, and topological derivatives for piezoelectric materials with small voids. SIAM JOurnal on Control and Optimization, 48(6):3925-3961, 2010.

[3] R. D. Mindlin. Influence of rotatory inertia and shear on flexural motions of isotropic, elastic plates. ASME Journal of Applied Mechanics, 18:31-38, 1951.

[4] A. A. Novotny, R. A. Feijóo, C. Padra, and E. Taroco. Topological derivative for linear elastic plate bending problems. Control and Cybernetics, 34(1):339-361, 2005.

[5] A. A. Novotny and J. Sokołowski. Topological derivatives in shape optimization. Interaction of Mechanics and Mathematics. Springer, 2013.

[6] E. Reissner. The effect of transverse shear deformation on the bending of elastic plates. ASME Journal of Applied Mechanics, 12:68-77, 1945.

[7] J. Sokołowski and A. Żochowski. On the topological derivative in shape optimization. SIAM Journal on Control and Optimization, 37(4):1251-1272, 1999. 